\title{
Photometric signatures of highly nonconvex and binary asteroids
}

\author{
J. Durech ${ }^{1}$ and M. Kaasalainen ${ }^{2}$ \\ 1 Astronomical Institute, Charles University Prague, V Holešovičkách 2, 18000 Prague, Czech Republic \\ 2 Observatory, PO Box 14, 00014 University of Helsinki, Finland
}

Received 7 February 2003 / Accepted 27 March 2003

\begin{abstract}
We present an analysis of synthetic lightcurves of highly nonconvex single or synchronous binary asteroids, paying particular attention to the connection between the available solar phase angles and the observable degree of asteroid nonconvexity. The measure of nonconvexity of an object is defined and computed for asteroids with known shapes. We discuss the possibility of recovering global nonconvexities or binary structure of an asteroid from its lightcurves. The importance of observations at high solar phase angles is emphasized. For main-belt asteroids only binary or bifurcated shapes can be resolved (at least in a qualitative sense) - topologically star-like asteroids can be modelled as convex bodies. Concavities can be revealed only if an asteroid is observed at sufficiently high solar phase angles $\left(\alpha>60^{\circ}\right)$ when shadowing effects from nonconvexities become important. This can be fulfilled only for near-Earth asteroids. Reconstruction of local topographic details is in practice precluded by the uncertainty of the light-scattering properties of the surface and the scarcity of very large phase angles.
\end{abstract}

Key words. minor planets, asteroids - techniques: photometric - methods: numerical

\section{Introduction}

Photometric lightcurves are a major source of information about asteroids. The method of asteroid lightcurve inversion developed by Kaasalainen et al. (2001) enables one to determine a convex model of an asteroid from its photometric diskintegrated lightcurves. The optimization procedure is robust and provides also a solution for the pole direction and rotation period. Such convex models describe the global shape of the asteroid well if the real shape is not very nonconvex.

Nonconvex inversion is technically quite feasible as well (Kaasalainen et al. 2001; Ďurech 2002), but so far real data have contained very little information on nonconvexities. To date, detailed models have been constructed from the lightcurves of almost one hundred asteroids (e.g., Kaasalainen et al. 2001, 2002a,b, 2003; Ďurech 2002; Slivan et al. 2003; Torppa et al. 2003), but so far convex models have explained all observations down to noise level, and no nonconvex model has produced a better fit to data. This has prompted us to find out exactly when one can get nonconvex information and what this information is.

In Sect. 2, we define the nonconvexity measure of an asteroid. In Sect. 3, we show that the discrepancy between original lightcurves and those generated by convex models increases with increasing phase angle and nonconvexity of an asteroid. Results are discussed in Sect. 4, Sect. 5 presents a summary.

Send offprint requests to: J. Durech, e-mail: durech@sirrah.troja.mff.cuni.cz

\section{Nonconvexity measure}

In order to describe the size of nonconvexity of an asteroid, we define the nonconvexity measure $\mathcal{V}_{\mathrm{nc}}$,

$\mathcal{V}_{\mathrm{nc}}=1-\frac{V}{V_{\mathrm{ch}}}$

where $V$ is the volume of an asteroid and $V_{\text {ch }}$ is the volume of its convex hull. The measure $\mathcal{V}_{\text {ch }}$ is dimensionless and equals the volume of concavities with respect to the volume of the convex hull divided by the volume of the convex hull. For convex bodies $\mathcal{V}_{\mathrm{nc}}=0$ and the more nonconvex the body is, the higher value of $\mathcal{V}_{\mathrm{nc}}$ it has up to the limiting value $\mathcal{V}_{\mathrm{nc}}=1$. The volume $V$ of a binary asteroid is equal to the sum of the volumes of its components.

Table 1 gives the values of $\mathcal{V}_{\mathrm{nc}}$ for some real asteroids with known shapes (from radar observations or spacecraft images) and for artificial bodies of simple shape (for scale reference). Note that the uncertainty of the shape models derived from radar observations (all asteroids in Table 1 except Gaspra, Ida and Eros) propagates to their $\mathcal{V}_{\text {nc }}$ values. For the real objects, we have for convenience already added two solar phase angle values of particular interest (when applicable). The angle $\alpha_{\min }$ will be defined below: it gives the lowest solar phase angle at which we may expect nonconvex surface features to show in the lightcurve data. The angle $\alpha_{\text {obs }}$, on the other hand, denotes the highest phase angle at which the object has been observed. As we can see, the two angles never really overlap each other for these targets. In two cases they just touch each other, but there were very few data points available for those solar phases. 
Table 1. The values of the nonconvexity measure $\mathcal{V}_{\mathrm{nc}}$ are given for real asteroids and asteroid models. Contact ellipsoids are two similar ellipsoids (with long semiaxes $a$ ) placed end-to-end in contact. Medium gap ellipsoids are separated at the distance $a$ and large gap ellipsoids at the distance $2 a$. The minimum phase angle $\alpha_{\min }$ for detection of nonconvexities and the maximum observed phase angle $\alpha_{\mathrm{obs}}$ are given for real asteroids.

\begin{tabular}{|c|c|c|c|c|}
\hline object & $\overline{\mathcal{V}_{\mathrm{nc}}}$ & $\begin{array}{c}\alpha_{\min } \\
{[\mathrm{deg}]}\end{array}$ & $\begin{array}{c}\alpha_{\mathrm{obs}} \\
\text { [deg] }\end{array}$ & Reference \\
\hline $1998 \mathrm{KY}_{26}$ & 0.025 & $>110$ & 28.1 & Ostro et al. (1999) \\
\hline Gaspra & 0.049 & 100 & 24.9 & Thomas et al. (1994) \\
\hline Bacchus & 0.078 & 110 & 96.5 & Benner et al. (1999) \\
\hline Castalia & 0.079 & 100 & 90.3 & Hudson \& Ostro (1994) \\
\hline Geographos & 0.111 & $>100$ & 60.5 & Hudson \& Ostro (1999) \\
\hline Toutatis & 0.121 & 110 & 51.8 & Hudson \& Ostro (1995) \\
\hline Golevka & 0.139 & 80 & 81.4 & Hudson et al. (2000) \\
\hline Eros & 0.150 & 60 & 59.2 & Zuber et al. (2000) \\
\hline Ida & 0.182 & 60 & 21.1 & Thomas et al. (1996) \\
\hline $\begin{array}{l}\text { contact } \\
\text { ellipsoids }\end{array}$ & 0.200 & - & - & \\
\hline Kleopatra & 0.364 & 50 & 25.1 & Ostro et al. (2000) \\
\hline $\begin{array}{r}\text { medium gap } \\
\text { ellipsoids }\end{array}$ & 0.385 & - & - & \\
\hline $\begin{array}{l}\text { large gap } \\
\text { ellipsoids }\end{array}$ & 0.500 & - & - & \\
\hline
\end{tabular}

\section{Inversion of synthetic lightcurves}

In order to study the behaviour of convex and nonconvex inversion for various types of shapes, we produced model shapes and their lightcurves. Random noise of $0.02 \mathrm{mag}$ was added to the simulations, and the lightcurves were processed in the same way as real data.

Asteroid shapes were approximated by a polyhedral model made of triangular facets. Photometric behaviour of the surface was described by Hapke's photometric function for a rough surface (Bowell et al. 1989) with parameters for a typical S-type asteroid: $w=0.23, g=-0.35, h=0.02, B_{0}=1.32$, $\bar{\theta}=20^{\circ}$ (Helfenstein \& Veverka 1989). However, the choice of the scattering model is not important here. The total amount of reflected light was computed as the sum of contributions from both visible and illuminated facets. Synthetic lightcurves (40 points uniformly spread over the rotation phase) were computed using a ray-tracing procedure. We generated lightcurves for phase angles from $1^{\circ}$ up to $120^{\circ}$ with the step of $10^{\circ}$, four lightcurves per each phase angle. Due to the fact that in real cases accurate absolute photometry is only seldom available, the lightcurves were treated as relative. We assumed the pole direction and the sidereal rotation period to be known - the typical pole direction uncertainty in real cases is about $\pm 5^{\circ}$ and its influence to our simulations can be neglected. The convex inversion of the lightcurve data set leads to a unique convex model of the original body.

The convex fit between the original lightcurves and lightcurves generated by the convex model is very good for low solar phase angles and the discrepancy increases with increasing phase angle. For very high phase angles or highly nonconvex shapes, the convex model is not sufficient and the lightcurves are not fitted well.

We have studied four different types of asteroid shapes: Gaussian random shapes, elongated Kleopatra-like shapes that we call "gnawed apples", binary objects and real asteroids with known shapes.

In each lightcurve set we found a solar phase angle such that lightcurves with lower phase angles were fitted well with the convex model and for lightcurves with higher phase angles the fit was unacceptable. We denoted this phase angle $\alpha_{\min }-$ the minimum solar phase angle at which the nonconvexity of an asteroid starts to play an important role in its lightcurves and the original shape cannot be approximated by a convex model. The definition of $\alpha_{\min }$ is not rigorous and the values of $\alpha_{\min }$ are determined subjectively from lightcurve fits. The uncertainty of $\alpha_{\min }$ is about $\pm 10^{\circ}$. Nevertheless the value of $\alpha_{\min }$ turned out to have a strong and easily describable correlation with the nonconvexity measure $\mathcal{V}_{\mathrm{nc}}$.

\subsection{Gaussian random shapes}

Gaussian random shapes can be easily generated in the way described in Muinonen 1996. We have generated four bodies with different $\mathcal{V}_{\text {nc }}$ (see Fig. 1). Gaussian random shapes with $\mathcal{V}_{\mathrm{nc}}>0.3$ consistently seem very unrealistic and probably do not represent shapes of real asteroids. Other types of objects with higher $\mathcal{V}_{\text {nc }}$ could be bifurcated or binary asteroids.

We can get quite good nonconvex inversion models of these shapes at high phase angles $\left(\alpha>60^{\circ}\right)$ where the nonconvex features are photometrically important. An example of this is shown in Fig. 2.
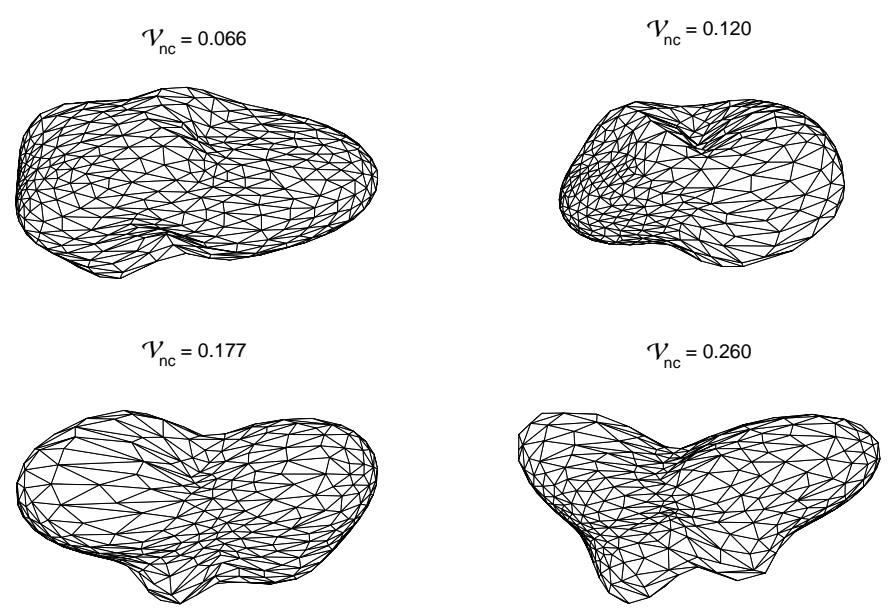

Fig. 1. Gaussian random shapes.

\section{2. "Gnawed apple" shapes}

The basic shape was an ellipsoid 2:1.4:1 and it was deformed to the rather artificial shape representing elongated bifurcated shapes (e.g. 216 Kleopatra). See Fig. 3. 

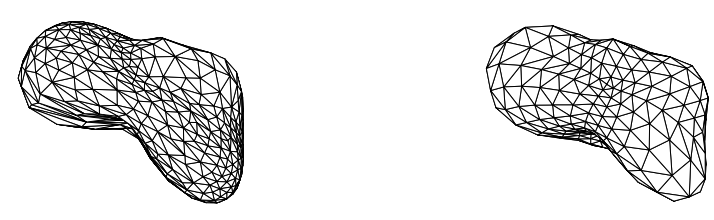

$\alpha=60$ deg
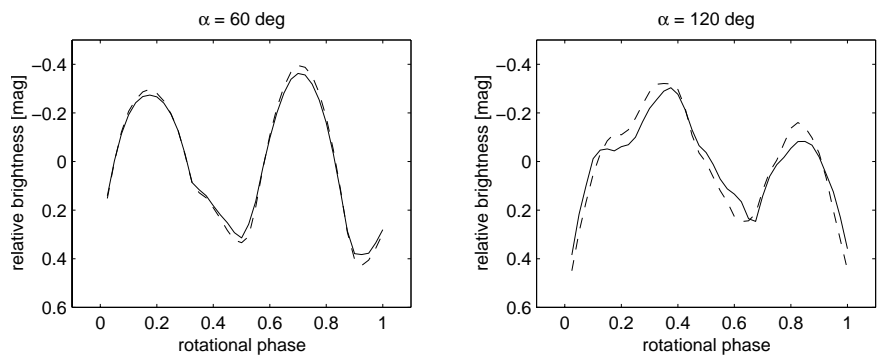

Fig. 2. An example of nonconvex inversion. The model (right) was derived from synthetic lightcurves (phase angles from $1^{\circ}$ to $120^{\circ}$ ) of the original object (left). Two typical lightcurves at phase angles $60^{\circ}$ and $120^{\circ}$ are shown. The discrepancy between the original lightcurves (solid curve) and the model lightcurves (dashed curve) increases with increasing phase angle.

$$
\mathcal{V}_{\mathrm{nc}}=0.013
$$

$$
\mathcal{V}_{\mathrm{nc}}=0.207
$$
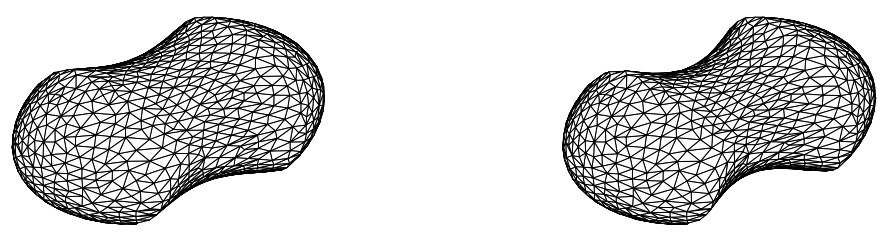

$$
\mathcal{V}_{\mathrm{nc}}=0.278
$$

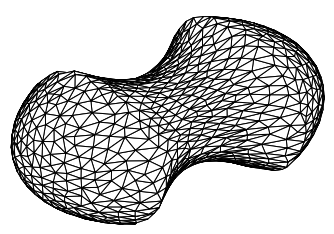

Our results show that contact binaries or binary asteroids only little separated can be modelled as single bodies with almost the same lightcurves. Therefore also the qualitative properties of the shape model (the conical asymmetry or brick-like shapes of convex models and the strong central narrowing of nonconvex models) should be taken into account in the interpretation of the inversion result. This method has picked out some main-belt asteroids as possible candidates for contact binaries (Kaasalainen et al. 2002a). To study this problem more closely, we generated four binary asteroids with increasing separation of their components (Fig. 4). All the binary systems have synchronous rotation and circular orbit and their components are of comparable size.
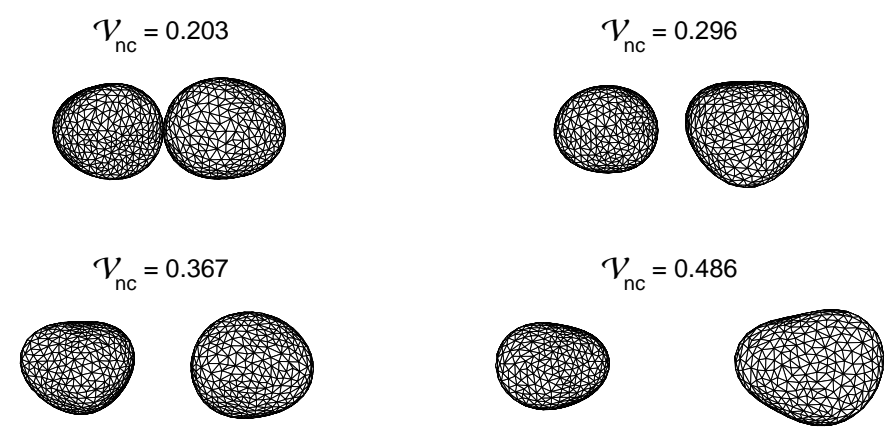

Fig. 4. Binary models with increasing separation of their components.

Convex inversion leads to a good fit of lightcurves but to a completely wrong shape (Fig. 5). A connected nonconvex inversion model clearly shows the binary nature, but the data may not be sufficient for concluding that the components are separate.

Although the convex "brick" is quite different in shape from the original binary asteroid, their lightcurves are very similar up to $\alpha=30^{\circ}$ and even for $\alpha=60^{\circ}$ do not differ much. The nonconvex model fits well the whole set of lightcurves up to $\alpha=120^{\circ}$ (Fig. 6).

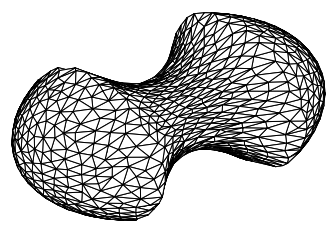

Fig. 3. "Gnawed apple" shapes.

\subsection{Binary asteroids}

Binary asteroids are considered to be an essential part of the whole asteroidal population. The clearest evidence of binary objects are two periods (for nonsynchronous rotation) and occultation/eclipsing features in their lightcurves. To date, most such photometric binary systems are NEAs for which the two components' mutual events are clearly distinguishable from the lightcurve background caused by the rotation of primary component (Pravec \& Hahn 1997; Mottola \& Lahulla 2000). Similar behaviour has been observed for the main-belt asteroid 90 Antiope (Michalowski et al. 2001), confirmed as a binary from adaptive optics observations (Merline et al. 2000) (contrary to the observed NEAs, the rotation seems to be synchronous for this object).

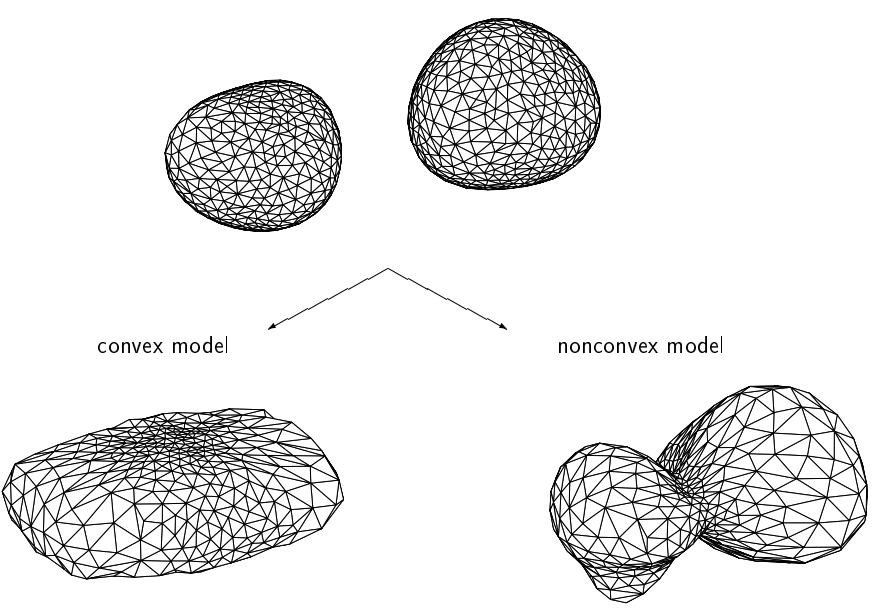

Fig. 5. Two models of a binary asteroid. 

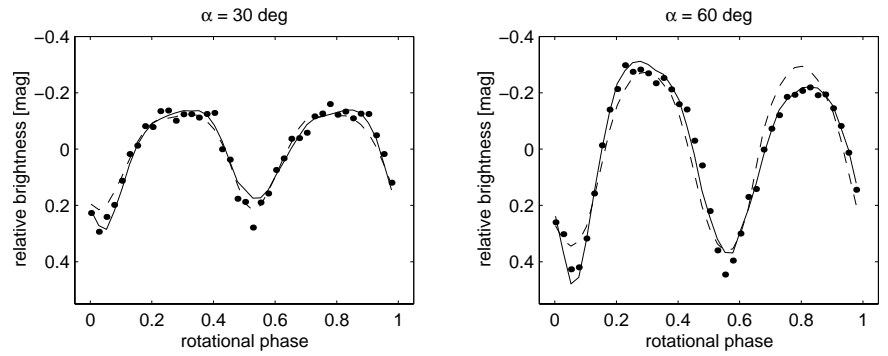

Fig. 6. Two typical lightcurves of the original binary system (points), the convex model (dashed curve) and the nonconvex model (solid curve) for solar phase angles $30^{\circ}$ and $60^{\circ}$.

\subsection{Real asteroids}

We also used ten asteroids with known shapes - from radar observations (1998 KY 26, Bacchus, Castalia, Geographos, Toutatis, Golevka, Kleopatra) or spacecraft images (Gaspra, Eros, Ida) - and generated their synthetic lightcurves. The asteroids cover a wide range of shapes - from nearly spherical $1998 \mathrm{KY}_{26}$ to very nonconvex Kleopatra. The $\alpha_{\min }$ values and the maximum observed phase angles $\alpha_{\text {obs }}$ are listed in Table 1. Except for Eros and Golevka, in all cases $\alpha_{\text {obs }}$ is significantly lower than $\alpha_{\min }$, and the two borderline cases seem to emphasize the rule by just falling short of suitable geometries of nonconvexity detection.

\section{Dependence of $\alpha_{\min }$ on $\mathcal{V}_{\mathrm{nc}}$}

There is a clear dependence between the nonconvexity measure of an object and the minimum solar phase angle $\alpha_{\min }$. This dependence is shown in Fig. 7 where all the studied objects are plotted. Notice that although the nonconvexity measure $\mathcal{V}_{\text {nc }}$ can be computed exactly, the phase angle $\alpha_{\text {min }}$ was not determined very precisely from the lightcurve fits. Thus the graph serves only as a first estimation of $\alpha_{\min }$ vs. $\mathcal{V}_{\mathrm{nc}}$ behaviour. Also, asteroids with the same $\mathcal{V}_{\text {nc }}$ can have different value of $\alpha_{\min }$ - for Gaussian shapes the values of $\alpha_{\min }$ are lower than for "gnawed apple" shape, i.e., Gaussian shapes are more "photometrically nonconvex" than bifurcated shapes with the same $\mathcal{V}_{\mathrm{nc}}$. The imaginary $\alpha_{\min }$ vs. $\mathcal{V}_{\mathrm{nc}}$ line shows the minimum phase angle needed to let us get quantitative nonconvex information on an object with given $\mathcal{V}_{\text {nc }}$, i.e., it should lie well above the line. In Table 1 and Fig. 7, $\alpha_{\min }$ is basically the last phase angle that can still just be explained with a convex model, so the really first phase angle that can give nonconvex information would be just a bit higher (cf. Eros and Golevka). Typically, at $\alpha_{\min }+10^{\circ}$ there is already a clear nonconvex signature - a nonconvex model fits the lightcurves significantly better than a convex model.

The three groups of asteroids - Gaussian random shapes, "gnawed-apple" shapes and binary models - create three lines in the plot that differ slightly in the slope and the vertical shift. This is probably due to the fact that bodies in each group have common type of shape and differ only in $\mathcal{V}_{\mathrm{nc}}$. Contrary to this, the real asteroids in the plot are more scattered than other groups - their shapes have no common characteristic.

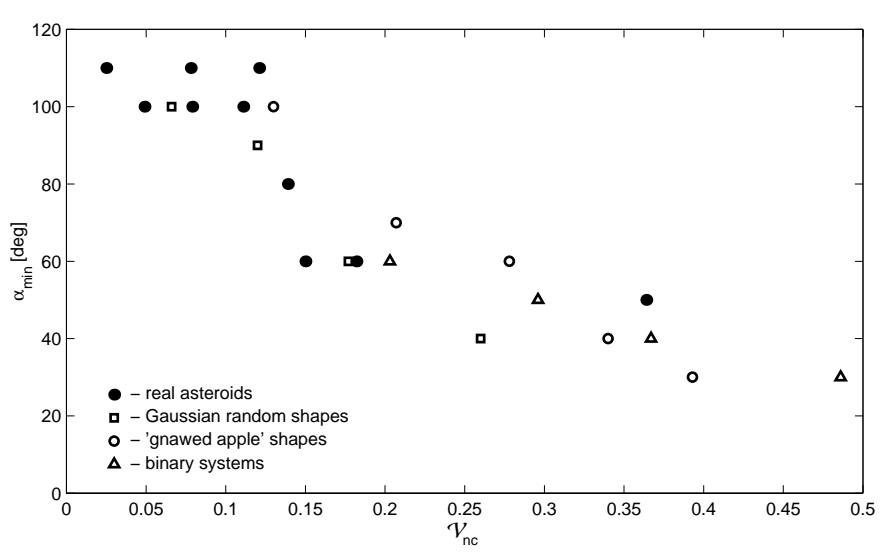

Fig. 7. Dependence of the minimum solar phase angle $\alpha_{\min }$ on the nonconvexity measure $\mathcal{V}_{\mathrm{nc}}$ for asteroids of various shapes.

Nevertheless, $\mathcal{V}_{\mathrm{nc}}$ seems to be a rather universal coarse-scale measure of nonconvexity in both visual and photometric sense.

We also estimated the maximum $\mathcal{V}_{\mathrm{nc}}$ values of asteroids 1627 Ivar, 3908 Nyx (Kaasalainen et al. 2003) and $60531993 \mathrm{BW}_{3}$ (Pravec et al. 1997a). For the real observation geometry we generated synthetic lightcurves of a Gaussian random shape. We increased the $\mathcal{V}_{\mathrm{nc}}$ values of our models until the discrepancy between the original lightcurves and lightcurves generated by a convex model was significant. For asteroids Ivar $\left(\alpha_{\text {obs }}=64^{\circ}\right)$ and Nyx $\left(\alpha_{\text {obs }}=39^{\circ}\right)$ the convex models fit well all the lightcurves even for the test shape with the highest $\mathcal{V}_{\mathrm{nc}}=0.26$. For the asteroid 6053 the phase angle reached the value $\alpha_{\text {obs }}=75^{\circ}$ and there are some nonconvex features in the lightcurves of the model with $\mathcal{V}_{\mathrm{nc}}=0.26$. This is consistent with the plot in Fig. $7-$ observations at $\alpha>70^{\circ}$ are necessary for detection of nonconvexities corresponding to $\mathcal{V}_{\mathrm{nc}} \sim 0.25$.

\subsection{The $\mathcal{V}_{n c}$ estimation of selected NEAs}

To date, there are 12 NEAs with the shape models determined from lightcurve inversion (excluding NEAs with space probe or radar shape models listed in Table 1). They are listed in Table 2. In all the 12 cases the convex model fits all observed lightcurves sufficiently well - none of the asteroids was observed at high enough phase angles or was sufficiently nonconvex to provide a significant nonconvex feature in its lightcurves. From the given maximum observed phase angles $\alpha_{\text {obs }}$ we can roughly place an upper bound on $\mathcal{V}_{\mathrm{nc}}$ values (within some \pm 0.05 or so) of these asteroids using the plot in Fig. 7. If their $\mathcal{V}_{\mathrm{nc}}$ values were significantly higher than our $\mathcal{V}_{\mathrm{nc}}^{\max }$ estimates given in Table 2, we should have detected it in their lightcurves.

\section{Conclusions}

The nonconvexity measure $\mathcal{V}_{\mathrm{nc}}$ has a clear connection with the minimum solar phase angle $\alpha_{\text {min }}$. For a given $\mathcal{V}_{\text {nc }}$ we are able to estimate the minimum solar phase angle below which a convex model describes photometric behaviour of the original body sufficiently well. Although the estimation of $\alpha_{\text {min }}$ from $\mathcal{V}_{\mathrm{nc}}$ is not very useful in practice, the inverse relation 
Table 2. The maximum observed solar phase angle $\alpha_{\mathrm{obs}}$ and the estimated (from the plot in Fig. 7) maximum nonconvexity measure $\mathcal{V}_{\mathrm{nc}}^{\max }$ (i.e. our inferred upper bound on the asteroid's true $\mathcal{V}_{\mathrm{nc}}$ ) are given for each asteroid.

\begin{tabular}{lcll}
\hline \hline \multicolumn{1}{c}{ asteroid } & $\alpha_{\text {obs }}[\mathrm{deg}]$ & $\mathcal{V}_{\mathrm{nc}}^{\max }$ & \multicolumn{1}{c}{ Reference } \\
\hline 1036 Ganymed & 55 & 0.35 & Hahn et al. (1989) \\
1580 Betulia & 59 & 0.3 & Wisniewski et al. (1997) \\
1627 Ivar & 64 & 0.25 & Chernova et al. (1995) \\
1862 Apollo & 86 & 0.15 & Harris et al. (1987) \\
1980 Tezcatlipoca & 67 & 0.25 & Kaasalainen et al. (2003a) \\
2100 Ra-Shalom & 42 & 0.45 & Pravec et al. (1998) \\
3103 Eger & 45 & 0.45 & Wisniewski (1987) \\
3199 Nefertiti & 60 & 0.3 & Pravec et al. (1997b) \\
3908 Nyx & 50 & 0.4 & Kaasalainen et al. (2003a) \\
5587 1990 SB & 70 & 0.25 & Koff et al. (2002) \\
6053 1993 BW 3 & 75 & 0.2 & Pravec et al. (1997a) \\
25143 1998 $\mathrm{SF}_{36}$ & 87 & 0.15 & Kaasalainen et al. (2003b) \\
\hline
\end{tabular}

(from $\alpha_{\min }$ to $\mathcal{V}_{\mathrm{nc}}$ ) is - if we have observations of an asteroid's lightcurves up to a solar phase angle of a certain value, we can give an estimation of how large nonconvexities of the original body we can reconstruct.

We have divided the range of the phase angle into three groups.

\section{Small phase angles $\alpha<30^{\circ}$}

Observations of main-belt asteroids are limited to solar phase angle $\alpha \sim 30^{\circ}$. Thus the shape models derived from such observations cannot provide nonconvex details. Shapes with $\mathcal{V}_{\mathrm{nc}}<0.35$ can be modelled as convex bodies and their lightcurves remain almost the same. Only for asteroids with $\mathcal{V}_{\mathrm{nc}}>0.35$ (binary or "exaggerated Kleopatra"-like bifurcated asteroids) can a nonconvex model be derived and their nonconvex nature partly revealed. If the convex inversion leads to a "brick-like" shape it may be an indication of the binary nature of the object and the nonconvex inversion can provide a two-lobe model (see Fig. 5).

\section{Moderate phase angles $\alpha<60^{\circ}$}

Near-Earth asteroids are often observed at phase angles higher than $30^{\circ}$. From such observations shape features corresponding to $\mathcal{V}_{\text {nc }} \sim 0.2$ can be reconstructed - global nonconvexities or binary nature of an object. The plausibility of the model depends on the observation geometry and the quality of data. Concrete shape models can be found in Kaasalainen et al. (2003).

\section{High phase angles $\alpha \leq 100^{\circ}$}

In order to reconstruct the asteroid shape in more detail, we need quality observations up to phase angle $\sim 100^{\circ}$ or more. Such observations are very rare.

With main-belt asteroids, we can obtain qualitative nonconvex models even when quantitative ones are hard to ascertain. There are some features that reveal the nonconvexity of original body, even if the convex model is quite sufficient - barrel-like or brick-like shapes are signs of binary nature of an asteroid, and large flat areas on the surface of a convex model are probably concavities.

Our simulations indicate that efforts should be made to obtain high phase angle NEA data that will allow the reconstruction of nonconvex details (so far we have analyzed only less than twenty good data sets of NEAs).

It is also useful to note in this context that there is one further practical limit to the topographic detail photometric data can provide, regardless of their accuracy and the available observing geometries. This limit is caused by the fact that the precise light-scattering properties of the surface are never very well known. While this does not affect the global-scale shape result (Kaasalainen et al. 2001, 2002b), it inevitably renders local features obscure. A simple proof of this is the fact that our global shape models always fit photometric data distinctively better than considerably more correct detailed space probe or radar-based shape models (those of the objects studied in Kaasalainen et al. 2001 as well as of 216 Kleopatra, 2063 Bacchus, and 4179 Toutatis). In fact, the photometric fit from the latter is consistently poor by our standards. This is not a paradox, it simply means that simple scattering models such as the Hapke one cannot describe real asteroid surfaces very accurately, particularly since the scattering properties can be expected to vary over the surface, and the scattering phenomena occur within a broad interval of length scales not explicitly taken into account in the scattering model (cf., e.g., the cratering density discussed in Kaasalainen et al. 2003).

In other words: when we construct a convex global shape, the detailed scattering properties are not very important, which is one of the reasons why the procedure is so robust (we know that the model shape is only an approximation of the real one, so the scattering uncertainty is about the same as just some noise). If we want to obtain a nonconvex model, the solar phase angle must be very high, and the nonconvexity must be of very global nature. Intermediate-scale or smaller nonconvexities are drowned in the observational noise, systematic errors, and the uncertainty of the scattering properties.

Acknowledgements. This work was supported by the research plan J13/98: 113200004 Investigations of the Earth and the Universe.

\section{References}

Benner, L. A. M., Hudson, R. S., Gstro, S. J., et al. 1999, Icarus, 139, 309

Bowell, E., Hapke, B., Domingue, D., et al. 1989, Application of photometric models to asteroids, in Asteroids II, ed. R. Binzel, T. Gehrels, \& M.S. Matthews (Tucson: University of Arizona Press), 524

Chernova, G. P., Kiselev, N. N., Krugley, Y. N., et al. 1995, AJ, 110, 1875

Ďurech, J. 2002, Icarus, 159, 192

Hahn, G., Magnusson, P., Harris, A. W., et al. 1989, Icarus, 78, 363

Harris, A. W., Young, J. W., Goguen, J., et al. 1987, Icarus, 70, 246

Helfenstein, P., \& Veverka, J. 1989, Physical characterization of asteroid surfaces from photometric analysis. In Asteroids II, ed. R. Binzel, T. Gehrels, \& M.S. Matthews

(Tucson: University of Arizona Press), 557

Hudson, R. S., \& Ostro, S. J. 1994, Science, 263, 940

Hudson, R. S., \& Ostro, S. J. 1995, Science, 270, 84

Hudson, R. S., \& Ostro, S. J. 1999, Icarus, 140, 369 
Hudson, R. S., Gstro, S. J., Jurgens, R. F., et al. 2000, Icarus, 148, 37

Kaasalainen, M., Torppa, J., \& Muinonen, K. 2001, Icarus, 153, 37

Kaasalainen, M., Torppa, J., \& Piironen, J. 2002a, A\&A, 383, L19

Kaasalainen, M., Torppa, J., \& Piironen, J. 2002b, Icarus, 159, 369

Kaasalainen, M., et al. 2003, Icarus, submitted

Koff, R. A., Pravec, P., Sarounova, L., et al. 2002, Minor Planet Bull., 29,51

Merline, W. J., Close, L. M., Shelton, J. C., et al. 2000, IAU Circ., 7503,3

Michalowski, T., Kwiatkowski, T., Kryszczynska, A., Colas, F., \& Michalowski, J. 2001, IAU Circ., 7757, 1

Mottola, S., \& Lahulla, F. 2000, Icarus, 146, 556

Muinonen, K. 1996, Earth Moon and Planets, 72, 339

Ostro, S. J., Pravec, P., Benner, L. A. M., et al. 1999, Science, 285, 557

Ostro, S. J., Hudson, R. S., Nolan, M. C., et al. 2000, Science, 288, 836
Pravec, P., \& Hahn, G. 1997, Icarus, 127, 431

Pravec, P., Wolf, M., Sarounova, L., Harris, A. W., \& Davies, J. K. 1997a, Icarus, 127, 441

Pravec, P., Wolf, M., Sarounova, L., et al. 1997b, Icarus, 130, 275

Pravec, P., Wolf, M., \& Sarounova, L. 1998, Icarus, 136, 124

Slivan, S., Binzel, R., Crespo da Silva, L., et al. 2003, Icarus, in press

Thomas, P. C., Veverka, J., Simonelli, D., et al. 1994, Icarus, 107, 23

Thomas, P. C., Belton, M. J. S., Carcich, B., et al. 120, 20

Torppa, J., Kaasalainen, M., Michalowski, T., et al. 2003, Icarus, submitted

Wisniewski, W. Z. 1987, Icarus, 70, 566

Wisniewski, W. Z., Michalowski, T. M., Harris, A. W., \& McMillan, R. S. 1997, Icarus, 126, 395

Zuber, M. T., Smith, D. E., Cheng, A. F., et al. 2000, Science, 289, 2097 